\title{
Review
}

Journal of Innate

Immunity

\section{Interplay between the Virus and Host in Rift Valley Fever Pathogenesis}

\author{
Kaori Terasaki ${ }^{a}$ Shinji Makino ${ }^{a-d}$ \\ ${ }^{a}$ Department of Microbiology and Immunology, ${ }^{b}$ Center for Biodefense and Emerging Infectious Diseases, ${ }^{C}$ UTMB \\ Center for Tropical Diseases and d Sealy Center for Vaccine Development, The University of Texas Medical Branch, \\ Galveston, Tex., USA
}

\section{Key Words}

Rift Valley fever · Viral pathogenicity · Innate immune responses

\begin{abstract}
Rift Valley fever virus (RVFV) belongs to the genus Phlebovirus, family Bunyaviridae, and carries single-stranded tripartite RNA segments. The virus is transmitted by mosquitoes and has caused large outbreaks among ruminants and humans in sub-Saharan African and Middle East countries. The disease is characterized by a sudden onset of fever, headache, muscle pain, joint pain, photophobia, and weakness. In most cases, patients recover from the disease after a period of weeks, but some also develop retinal or macular changes, which result in vision impairment that lasts for an undefined period of time, and severe disease, characterized by hemorrhagic fever or encephalitis. The virus also causes febrile illness resulting in a high rate of spontaneous abortions in ruminants. The handling of wild-type RVFV requires high-containment facilities, including biosafety level 4 or enhanced biosafety level 3 laboratories. Nonetheless, studies clarifying the mechanisms of the RVFV-induced diseases and preventing them are areas of active research throughout the world. By primarily referring to recent studies using several animal model systems, protein expression systems, and specific mutant viruses, this review describes the current knowledge
\end{abstract}

about the mechanisms of pathogenesis of RVF and biological functions of various viral proteins that affect RVFV pathogenicity.

(c) 2015 S. Karger AG, Basel

\section{Introduction}

Rift Valley fever virus (RVFV) is a zoonotic arbovirus and a pathogen of RVF which is endemic in sub-Saharan African countries [1]. The disease is characterized by a high rate of spontaneous abortion in ruminants. Adult ruminants are relatively resistant to RVFV infection, while the mortality rate in newborn lambs is nearly $100 \%$ [1]. Humans infected with the virus show acute influenza-like symptoms with fever, headache, and body pain. In some cases, patients develop neurological disorders and ocular disease, or die due to hemorrhagic fever and thrombosis [2]. RVFV is mainly transmitted by mosquito bite and, to a lesser extent, direct contact with materials from infected animals. There is concern that RVFV can be introduced into nonendemic countries by infected travelers, animals and mosquitoes; moreover, naturally occurring mosquito populations, in some nonendemic countries, including the US, support RVFV replication.

RVFV belongs to the genus Phlebovirus, family Bunyaviridae, and carries single-stranded tripartite RNA seg-

Dr. Kaori Terasaki

Department of Microbiology and Immunology, The University of Texas Medical Branch 4.162 Medical Research Building, 301 University Boulevard Galveston, TX 77555-1019 (USA)

E-Mail katerasa@utmb.edu 
Fig. 1. Schematic diagram of RVFV antigenomic RNAs. Three viral RNA segments and viral proteins encoded in each segment are shown. The five in-frame translation initiation codons present in the pre-Gn region of $\mathrm{M}$ segment are illustrated by five short vertical lines. Viral RNA segments are not depicted according to their sizes.

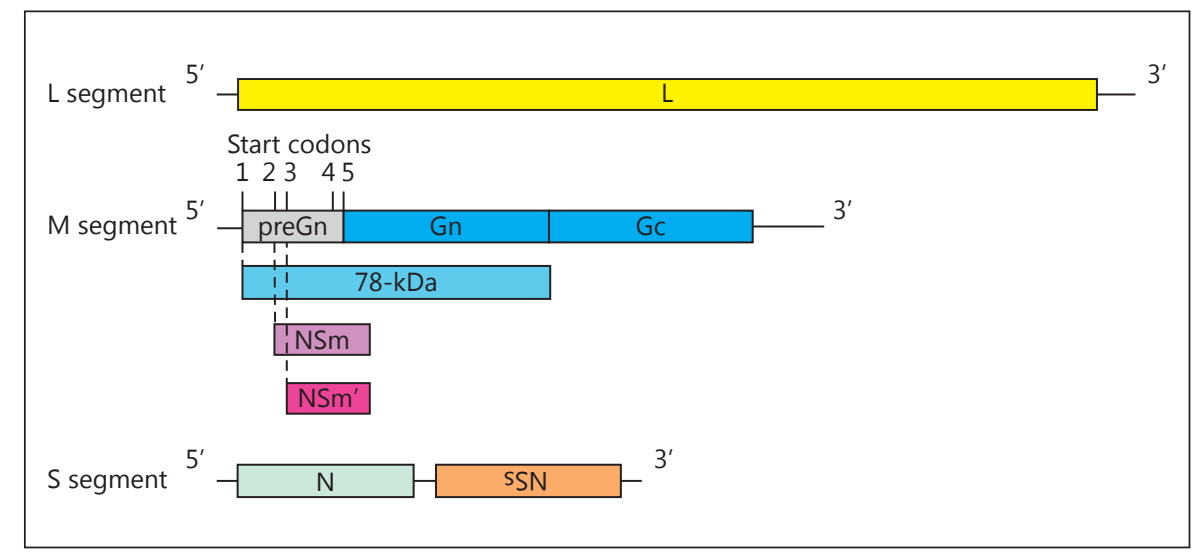

ments designated $\mathrm{L}, \mathrm{M}$, and $\mathrm{S}$ of negative or ambisense polarity (fig. 1) [2]. The antiviral-sense L RNA encodes the RNA-dependent RNA polymerase (L protein). The antiviral-sense M RNA encodes two envelope glycoproteins, Gn and Gc, and two accessory proteins, NSm and the $78-\mathrm{kDa}$ protein. The $\mathrm{S}$ segment uses an ambisense strategy for gene expression, and a nonstructural protein, NSs, is translated from the mRNA that is transcribed from the antiviral-sense $\mathrm{S}$ segment, whereas $\mathrm{N}$ protein is produced from the mRNA that is transcribed from the viral-sense $S$ segment. RVFV is an enveloped virus having an icosahedral symmetry and containing the segmented viral RNA genomes, L, N, and envelope glycoproteins, and several host proteins [3].

This review provides an overview of RVFV pathogenesis in animal model systems and summarizes our current knowledge about biological functions of viral proteins that affect RVFV pathogenesis.

\section{Pathogenesis of RVFV: Tissue Tropism and Host Responses in Experimental Models for RVFV Infection}

\section{Early Stage of Infection}

The pathogenesis of RVFV has been investigated with several different animal models, including ruminants, rodents, and nonhuman primates (for details, see review [2]). Although susceptibility to RVFV is dependent on animal species and strains, most of the susceptible animals develop acute hepatic and neurological diseases, which are also observed in human RVF cases $[1,2,4]$. Clinical outcomes of RVFV infection in these experimentally infected animals can vary significantly depending on the route of infection. Inoculation of mice by the intra- peritoneal (i.p.) route, compared to subcutaneous (s.c.) or intradermal (i.d.) inoculation, results in efficient virus replication in the liver and leads to the development of severe diseases $[5,6]$. Conversely, i.p. inoculation of the virus sometimes does not cause detectable viremia in sheep [2]. Intranasal (i.n.) inoculation, and aerosol exposure have caused efficient virus replication in mouse brains [7-10] and even in an RVFV-resistant rat strain [11]. The i.d. and s.c. inoculation routes also have been used to mimic the natural route of virus transmission through a mosquito bite.

As an arbovirus, RVFV is injected into mammalian hosts along with a mosquito salivary component. Mosquito saliva contains anti-inflammatory molecules to maintain blood flow during feeding. This effect of saliva sometimes makes the environment more permissive for arthropod-borne viruses and helps virus replication (for review, see [12]). In the case of RVFV, infection in mice has been reported to be facilitated by mosquito saliva. Mice coinjected with RVFV and mosquito saliva showed higher virus titers in target organs and shorter survival times than did the mice inoculated with RVFV alone [5]. RVFV derived from mammalian and insect cells differs in its ability to infect goat monocyte-derived dendritic cells (DCs) [13]. The virus from insect cells infects more efficiently than that from mammalian cells and showed a faster viremia peak in the infected goat, indicating that DCs may have a role in controlling RVFV pathogenesis.

In infected mammalian hosts, the virus first encounters macrophages and DCs, the first line of host defense, in local tissue exposed to the incoming virus. Although phagocytes can actively intake any invaders, RVFV is able to replicate in macrophages in vitro and in vivo $[6,13-$ 18]. In human monocyte-derived macrophages, secretion 
of tumor necrosis factor (TNF)- $\alpha$, interferon (IFN)- $\beta$, and IFN- $\alpha 2$ was induced by infection of a virus lacking NSs, but not by wild-type RVFV, indicating that NSs inhibited the secretion of these proteins in wild-type RVFVinfected macrophages [17]. Other proinflammatory cytokines were under the detectable level even after the infection of NSs-deletion virus [17]. These results may indicate that RVFV-infected macrophages are unable to induce efficient proinflammatory responses.

DCs are also an important target of RVFV. The virus uses DC-SIGN, a C-type lectin expressed in immature DCs, as an entry receptor [19]. While, the role of DCSIGN in an innate immune response is not well characterized, some viruses target DC-SIGN for efficient dissemination within the host. Hepatitis $\mathrm{C}$ virus internalized into DCs through DC-SIGN escaped from lysosomal degradation, implying that the DCs protected the virus as a reservoir [20]. HIV-1 captured by DCs through DC-SIGN retained long-term infectivity and was efficiently transmitted to CD4+ T cells [21]. Currently, it is unclear whether DC-SIGN serves another role(s), other than that of a receptor for RVFV, in RVFV pathogenicity. Several studies have explored a role(s) of DCs and macrophages in RVFV pathogenesis. In type I IFN-deficient mice inoculated with attenuated RVFV lacking the NSs gene by the i.p. route, virus replication was detected in macrophages, DCs, and granulocytes. Depletion of both macrophages and DCs in the mice resulted in slower viral dissemination, decrease in RVFV titers in blood, and prolonged survival, possibly meaning that these immune cells play a role in increased pathogenicity [6]. Ermler et al. [18] demonstrated that bone marrow-derived murine conventionalDCs and macrophages expressed IFN- $\alpha$ through the mitochondrial antiviral signaling protein signaling pathway in response to infection from RVFV lacking the NSs gene. The absence of mitochondrial antiviral signaling protein resulted in an increase in virus titers in conventional DCs, but not in macrophages, which may mean that these immune cells utilize different defense mechanisms against RVFV infection. The authors also speculated that the virus may use different mechanisms of entry into these cells, resulting in differing responses to RVFV infection.

\section{Middle Stage of Infection (Hepatic Phase)}

Live imaging of IFN receptor-knockout mice infected with RVFV carrying a luciferase gene, in place of the NSs gene, was used to examine the tissue tropism of RVFV [6]. After s.c. inoculation of this mutant virus, light emission first appeared in draining lymph nodes, a contact site of the lymph system and blood vessels. Subsequently, the virus entered the blood and underwent replication in a broad range of organs, including the liver, spleen, kidneys, pancreas, lungs, and other organs $[5,6,14,22]$. RVFV utilizes heparin sulfate, which is abundantly expressed on most cell types, to infect a broad range of cell types [23].

The liver has been described as a main target of RVFV, and abundant virus antigens have been found in liver isolated from RVFV-infected animals regardless of the infection route $[4,8,9,22,24,25]$. The livers of infected animals have shown massive hepatic necrosis, leading to inflammation with infiltration of neutrophils $[8,9,22]$. Apoptosis of hepatocytes was also observed in the liver during the hepatic phase $[22,26]$. Proinflammatory and antiapoptotic genes were activated, while proapoptotic genes were also upregulated in the livers of mice infected with virulent RVFV [27]. Interestingly, a recent ultrastructural study of RVFV-infected mice revealed the rare observation of mature virus particles in the liver during the course of infection, which may mean that the virus undergoes abortive replication in hepatocytes [26]. During the late stage of infection, liver repair was observed in mice inoculated with a lethal dose of virus $[14,22,26]$. At this stage, a sharp decrease in cytokine and chemokine production was observed [25].

The roles of cytokines and chemokines in RVFV pathogenesis have been examined in several studies. Infection of virulent RVFV in mice and goats has resulted in the activation of IL-12, which induces IFN- $\gamma$ production by NK cells and a Th1 response $[13,25,27]$. This reaction serves to limit viruses, while it also causes immunopathology. The livers of mice infected with a live-attenuated RVFV MP-12 were histologically normal, although infectious RVFV was isolated from the organ. On the other hand, virulent RVFV infection caused severe liver damage, including hepatocellular degeneration and necrosis. MP-12 infection caused a minimal cytokine response, whereas virulent RVFV infection elicited the systemic production of cytokines and chemokines, including IL-1 $\alpha$, IL-12, G-CSF, KC, and MCP-1. These results indicated a correlation between expression levels of cytokines/chemokines and tissue pathology in mice [25]. It is possible that the uncontrollable inflammation and cytotoxicity of the innate immune response caused lethal liver damage in wild-type RVFV-infected animals. IL-10, an anti-inflammatory cytokine, was also upregulated in mice infected with virulent RVFV [27]. IL-10 protects the host from immunopathology by ameliorating excessive Th1 and CD8 T cell responses; however, at the same time, it 
limits the clearance of pathogens [28]. In human RVF cases, IL-10 was elevated in fatal cases in comparison to its level in nonfatal cases [17]. As adaptive immunity is critical for RVFV clearance from the liver and brain [29], induction of cytokines which stimulate adaptive immune responses is important for recovery from the disease. The proper control of these cytokines, including IL-12 and IL10, which decide the balance of immunopathology and virus clearance, may be the key to recovery from RVFVinduced disease.

\section{Late Stage of Infection}

Although neurological disorders are rare in humans, many highly susceptible animal models show neurological symptoms late in the course of RVFV infection [2]. In mice inoculated with RVFV via the s.c. route, multiple cell types, including neurons and neuroglial cells, were positive for the virus antigens in most regions of the brain, including the brain stem, olfactory tract, and spinal cord [22]. Abundant virus particles budding and accumulating in multiple cell types of the central nervous system (CNS) were found after electromicroscopic analysis [26]. Histopathological analyses of RVFV-infected animals showed infiltration of inflammatory cells, neuronal necrosis, and neurophagia in multiple regions of the brain, indicating meningitis and encephalitis $[7,8,22,24]$. After s.c. inoculation of RVFV into the mice, virus antigens were found in olfactory neurons located in the olfactory mucosa, demonstrating that these neurons are an RVFV target and implying the possibility that the virus invades the brain subsequent to infection of olfactory nerves [22]. Olfactory neurons were also found positive for RVFV antigen in mice challenged by the aerosol and intranasal routes $[9,10]$.

Other studies have put forth the possibility that the virus might enter the CNS through the blood-brain barrier (BBB) or cerebrospinal fluid (CSF) barriers. Coinjections of RVFV with salivary gland extract via the i.d. route increased the mortality rates in mice and virus titers in several organs, including the brain cortex [5]. The author concluded that mosquito saliva might have indirectly facilitated viral invasion of the brain by modulating the permeability of the BBB. As mosquito saliva contains antiinflammatory molecules, it may be possible that it modulates the host innate response which allows efficient RVFV replication at an initial stage of infection and also affects subsequent cytokine secretion patterns, which may affect BBB permeability [12]. CSF barriers in the choroid plexus, where CSF is produced, are not completely protected by the $\mathrm{BBB}$ due to a fenestrated endothelial

Virus and Host Interplay in RVFV Pathogenesis cell layer. An example of viral infection of the CSF barrier is echovirus 30 , a human enterovirus causing meningitis, which can directly infect choroid plexus epithelium cells, a component of the CSF barrier [30].

Although interaction of RVFV with CSF barriers is unknown, the presence of RVFV in CSF has been reported in humans and animals [24, 31]. However, this occurrence may depend on animal species, infection route, time of sample collection, or sensitivity of assay. In African green monkeys or marmosets challenged by the aerosol route, no virus was detected in the CSF, despite the high levels of virus in the brain and spinal cord [7]. CSF collected from calves challenged by s.c. RVFV inoculation contained high titers of virus [24]. In human cases, RVFV was isolated in cell culture from $1.8 \%$ of the CSF samples from patients, whereas RT-PCR amplified RVFV-specific RNA from $13.6 \%$ of the CSF samples [31]. Clarification of the mechanisms of RVFV invasion into the CNS requires further studies.

\section{Viral Factors That Affect RVFV Pathogenesis}

To identify virus proteins that affect viral virulence/ pathogenesis, initially the pathogenicity of several naturally occurring RVFV mutants of known genetic sequence was tested. An attenuated MP-12 strain was developed by serial passage of the ZH548 strain, which was isolated from human serum during the 1977 outbreak in Egypt, in the presence of the mutagen 5-fluorouracil with subsequent plaque cloning and amplification (for review, see [32]). MP-12 has total 23 nucleotide changes, including 9 nucleotide changes leading to 9 amino acid changes, in the viral genome compared to its parent strain. Analysis of the virulence properties of reassortant viruses between the virulent strain and MP-12 showed that the virulence characteristics in the mouse were under polygenic control [33]. Another study demonstrated that chimeric viruses carrying the $L$ and $M$ segments of virulent ZH548 and the S segment of MP-12 were slightly attenuated, but still retained virulence in mice [34]. Clone 13 is another attenuated virus isolated by plaque cloning of the $74 \mathrm{HB} 59$ strain, and carries a deletion of $69 \%$ of the NSs gene [35]. Clone 13-derived mutant virus carrying the $\mathrm{S}$ segment of ZH548 was virulent in mice, suggesting that the $S$ segment controls the virulence [36]. These results indicated that each segment contributed to the virulence of the virus.

Table 1 summarizes the biological functions of RVFV proteins that affect viral pathogenicity and virulence. 
Table 1. Role of RVFV proteins in virus replication and virulence

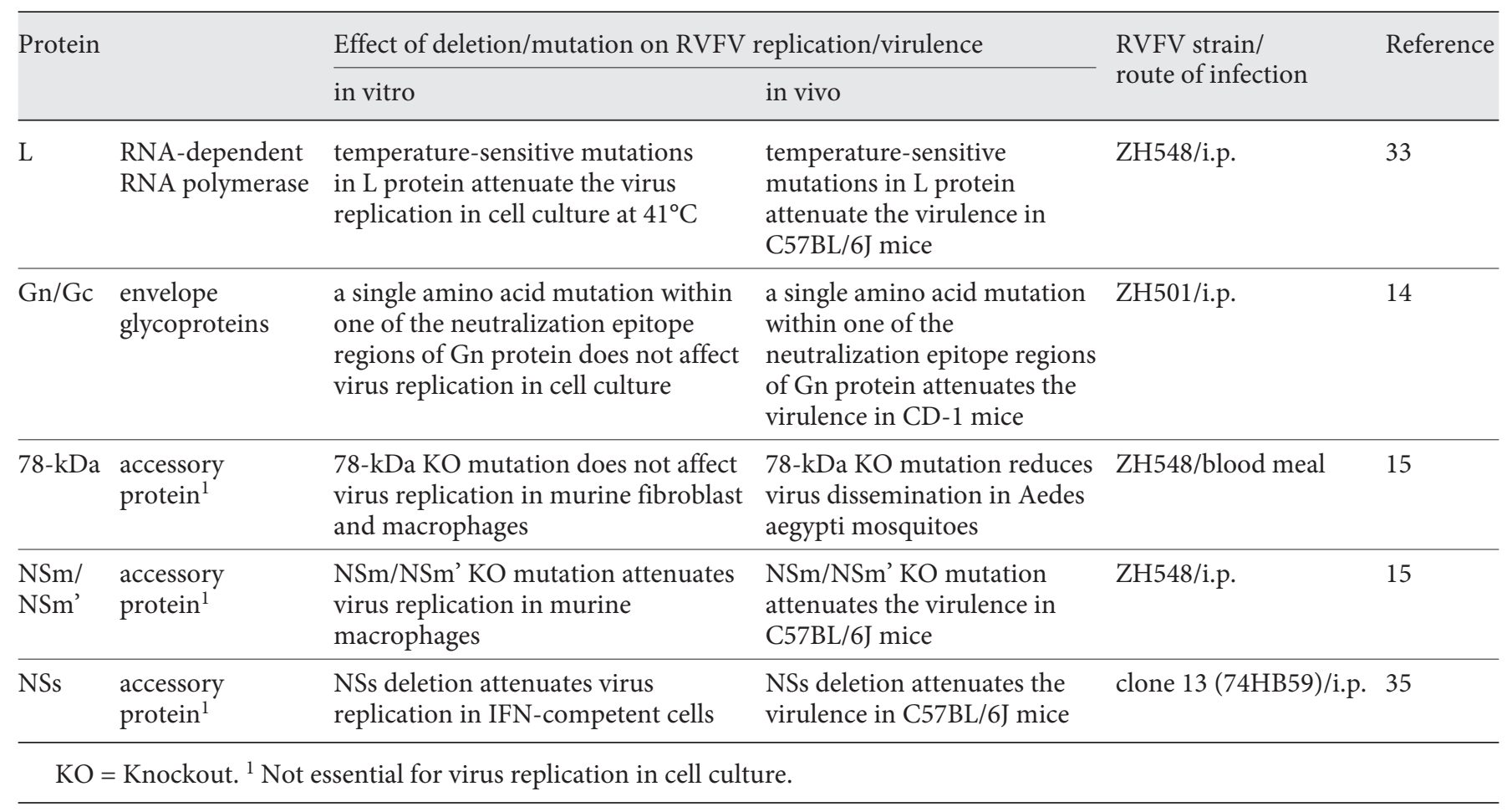

\section{NSs Protein}

RVFV NSs protein is a nonstructural protein with a molecular weight of $31 \mathrm{kDa}$. Although NSs is not essential for RVFV replication in cultured cells [37], it is required to establish productive infection in mice $[29,38]$. NSs has multiple functions (reviewed in [32]). NSs localizes both the nucleus and cytoplasm in infected cells, as well as in expressed cells, and forms filamentous structures in the nucleus. One of the important biological functions of NSs in RVFV pathogenesis is its inhibition of IFN- $\beta$ gene expression. NSs binds to SAP30, a subunit of the corepressor complex, and reinforces the interaction of the complex to repress IFN- $\beta$ mRNA synthesis. In addition, NSs binds to p44 and p62, subunits of transcription factor TFIIH, and inhibits host mRNA synthesis. Several in vitro studies using NSs deletion virus demonstrated that NSs inhibits the expression of host antiviral proteins. Infection of RVFV lacking an NSs gene induced the synthesis of IFN- $\alpha$, IFN- $\beta$, and TNF- $\alpha$, whereas induction of these proteins was not observed in parental RVFV-infected cells $[17,18,37,38]$.

Type I IFN plays a critical role in protecting the host from RVFV infection in animal models. A recent study demonstrated that IFN-induced transmembrane protein
2 and 3 inhibit virus replication at an early stage of infection after viral entry [39]. In type I IFN receptor-knockout mice, even attenuated RVFV lacking the NSs protein causes lethal infection $[6,40]$. Most of the sub-Saharan RVFV strains were less pathogenic than were Egyptian strains in RVFV-susceptible Wistar-Furth rats. These Egyptian strains were relatively more resistant to rat type I IFN than were the sub-Saharan RVFV strains, implying that type I IFN expression in the rat reduced the pathogenicity of the sub-Saharan RVFV strains [41]. Detailed time-course monitoring of IFN expression in rhesus monkeys infected with virulent RVFV showed that a delayed IFN-a response contributed to a lethal outcome, indicating the importance of type I IFN in very early stages of infection [42]. NSs inhibits type I IFN production early in infection and facilitates virus replication and dissemination.

Another known function of NSs is its inhibition of eIF $2 \alpha$ phosphorylation by promoting degradation of dsRNA-dependent protein kinase (PKR) through the proteasome pathway [32]. Activation of PKR by RVFV infection causes eIF2 $\alpha$ phosphorylation, resulting in translational arrest at a translation initiation step. Furthermore, host transcription suppression by NSs limits 
new synthesis of proteins, including eIF $2 \alpha$. NSs protects eIF $2 a$ from phosphorylation by PKR degradation and allows the virus to translate viral proteins.

Clone 13 virus carrying a deletion of $69 \%$ of the NSs gene did not cause lethal infection in mice, but did so in PKR-deficient mice [38], indicating that PKR plays an important role in protecting mice from RVFV infection. PKR is an IFN-stimulated gene and mediates several antiviral functions. In response to virus infection, PKR mediates apoptosis by eF $2 \alpha$ phosphorylation and NF-kB activation [43]. In addition, PKR is required for IFN- $\beta$ secretion in response to some virus infections; in the case of Semliki Forest virus, rotavirus, and West Nile virus, secretion of IFN was significantly lower in infected mouse embryonic fibroblasts derived from PKR knockout mice compared to those derived from wild-type mouse embryonic fibroblasts ([44] and references within). Interestingly, in PKR-deficient cells infected with Semliki Forest virus, IFN- $\beta$ mRNA were produced but these mRNA lacked a poly $(\mathrm{A})$ tail, suggesting the involvement of PKR in the regulation of IFN mRNA processing/stability [45]. In contrast, PKR was not required for type I IFN synthesis in the case of Sendai or influenza virus infections [45]. It would be of interest to know whether NSs affects posttranscriptional regulation of IFN- $\beta$ mRNA that has escaped from the NSs-induced suppression of transcriptional initiation. In summary, NSs protein inhibits the expression of host genes, including IFN and others with anti-viral functions, to establish infection in mammalian hosts.

RVFV carrying NSs forms clear plaques and induces a strong clear cytopathic effect (CPE) in infected VeroE6 cells, whereas RVFV lacking NSs forms turbid plaques and shows low levels of CPE [37], demonstrating that NSs contributes to the cytotoxicity of the virus. However, the PKR degradation function of the NSs alone does not determine the severity of the virus-induced CPE, because RVFV carries an NSs mutant that does not promote PKR degradation, but retains other functions, e.g. inhibition of synthesis of host transcripts and IFN- $\beta$ mRNA, and its parental virus formed similar CPEs in infected cells [46]. NSs-induced host transcriptional suppression may be responsible for the NSs-mediated cytotoxicity, whereas experimental evidence to support this expectation is missing.

RVFV lacking a functional NSs still induces fatal disease in type I IFN-KO mice and PKR-KO mice [38]. Furthermore, MP-12 carrying a fully functional NSs is highly attenuated. These data indicate that other virus proteins also contribute to virus pathogenesis.

Virus and Host Interplay in RVFV

Pathogenesis

\section{NSm and NSm' Proteins}

The $M$ segment has 5 in-flame AUGs and expresses $78-\mathrm{kDa}, \mathrm{NSm}$ and $\mathrm{Gn} / \mathrm{Gc}$ proteins from $1 \mathrm{st}$, 2nd, and 4th AUG, respectively, due to its leaky scanning mechanism (fig. 1). These 5 AUGs are highly conserved among RVFV strains [47]. A recent study revealed that a truncated NSm protein called NSm' is expressed from the 3rd AUG; NSm' lacks the N-terminal 38 amino acids of NSm and is predominantly expressed compared to NSm in infected Vero E6 cells, when both the 2nd and 3rd AUG are intact [15]. The C-terminal region of NSm, which contains a basic amino acid cluster and a putative transmembrane domain, targets the protein to the mitochondorial outer membrane [48]. Since the C-terminal region is common in NSm and NSm', NSm' is also localized to mitochondria [15].

$\mathrm{NSm}$, a $14-\mathrm{kDa}$ nonstructural protein, is not required for RVFV replication in cultured cells [49]. By targeting the mitochondrial outer membrane, NSm suppresses apoptosis that is induced by RVFV replication or staurosporine in the absence of other RVFV proteins [16, 48]. Thus far, two studies have revealed the importance of NSm in RVFV pathogenicity in rodents. Bird et al. [50] first reported that the wild-type RVFV stain lacking $78-\mathrm{kDa} / \mathrm{NSm} / \mathrm{NSm}$ ' caused reduced viral virulence in rats. Subsequently, Kreher [15] reported the generation of three RVFV mutants, each of which lacks NSm, NSm', or both NSm and NSm'. In 3-week-old mice challenged with the virus by i.p. inoculation, deletion of NSm alone or NSm' alone did not have any significant impact on viral virulence, whereas the mutant virus lacking expression of both NSm and NSm' was strongly attenuated. This $\mathrm{NSm} / \mathrm{NSm}$ ' deletion mutant replicated efficiently in a mouse fibroblast cell line, whereas its titers were significantly reduced in murine macrophages [15]. The mechanism of reduced virulence of RVFV lacking both NSm and NSm' expression is currently unknown, and yet the inefficient replication of the virus in macrophages may contribute to its reduced virulence.

\section{8-kDa Protein}

$78-\mathrm{kDa}$ protein is translated from the 1st AUG of $\mathrm{M}$ mRNA (fig. 1). Although 78-kDa protein is not essential for virus replication in cell culture [49], RVFV lacking 78-kDa, NSm and NSm' proteins lost its infectivity to mosquitoes, indicating the importance of these proteins in this insect $[15,51]$. Virus lacking $78-\mathrm{kD}$ a protein showed low dissemination rates in mosquitos, indicating that $78-\mathrm{kDa}$ protein is a determinant of virus dissemination in the mosquito. $78-\mathrm{kDa}$ protein is incorporated into virions released from cells of insect origin, but not in virions released from

J Innate Immun 2015;7:450-458 
cells of mammalian origin [52]. Immune electron microscopic analysis using an antibody which recognizes only 78 - $\mathrm{kDa}$ protein confirmed the presence of the 78 -kDa protein on the surface of virions [52]. Two predicted glycosylation sites in $78-\mathrm{kDa}$ protein are both glycosylated ([52] and references within). These observations indicate that a majority of the $78-\mathrm{kDa}$ protein is exposed to the endoplasmic reticulum lumen within the cells and exposed to the outside of the viral envelope.

Nfon et al. [13] reported that insect cell-derived RVFV (IN-RVFV) and mammalian cell-derived RVFV (MAMRVFV) had different effects in goats after s.c. challenge. Although these two viruses showed similar viremia titers, IN-RVFV showed an earlier peak titer. The reduction of CD172a+ cells (monocytes and DCs) was more significant in IN-RVFV-infected goats, and IN-RVFV was able to infect PBMCs and monocyte-derived macrophages more efficiently than MAM-RVFV. In addition, INRVFV induced a greater IL- 12 production than did MAM-RVFV. Their data suggested that efficient INRVFV replication in these immune cells helped in the dissemination of IN-RVFV, resulting in a faster peak of viremia titers of IN-RVFV. As described above, it is possible that IN-RVFV, but not MAM-RVFV, carried the $78-\mathrm{kDa}$ protein [52]. Although the presence of the 78$\mathrm{kDa}$ protein may not be the only difference between INRVFV and MAM-RVFV, data shown in the paper of Nfon et al. [13] indicate a possibility that $78-\mathrm{kDa}$ contributes to the increased infectivity of the virus to certain immune cells.

Although 78-kDa protein is not essential for virus replication in mammalian cells, competition-propagation assays using Vero E6 cells show that RVFV expressing $78-\mathrm{kDa}$ protein had a selective advantage over the virus lacking this protein [49]. Also, serial passage of the RVFV lacking $78-\mathrm{kDa}$ protein expression in Vero E6 cells resulted in production of virus carrying a new AUG to express $78-\mathrm{kDa}$ protein [15]. These results may indicate a beneficial role of $78-\mathrm{kDa}$ in virus replication.

\section{Other Virus Proteins}

Morrill et al. [14] found that a stock of wild-type RVFV ZH501 strain obtained from a patient during the 1977 outbreak of RVFV in Egypt contained two major viral populations, ZH501-M847-A and ZH501-M847-G, which carried $A$ residue and $G$ residue, respectively, at position 847 in the M segment (M847), resulting in one amino-acid difference at position 277 of the Gn protein; M847 was the only detectable main nucleotide substitution in the virus stock. Sequence analysis showed that 38 out of 39 strains emerging in Africa and Saudi Arabia had $\mathrm{A}$ at M847, possibly indicating that ZH501-M847-A-type virus represents the major virus population. The growth kinetics of these viruses in cultured cells was similar. The i.p. inoculation of recombinant virus rZH501-M847-A carrying Glu 277 killed most of the mice within 8 days, whereas recombinant rZH501-M847-G carrying Gly 277 had attenuated virulence in mice. These data clearly showed that single nucleotide substitution at M847 affects RVFV virulence in mice; however, the reason for the attenuation of ZH501-M847-G remains unclear. rZH501-M847-G virus quickly elicited neutralizing antibody while rZH501-M847-A virus showed a delayed antibody production compared to that with rZH501M847-G. This implied that rZH501-M847-A suppressed the host immune response at an early stage of infection.

MP-12, which was generated by serial plaque passages of ZH548 strain 12 times in MRC-5 cells, is attenuated and temperature sensitive ( $t s$ ) [33]. Although both $\mathrm{L}$ and $M$ segments are responsible for the $t$ s phenotype, $t s$ mutation in $\mathrm{M}$ segment did not affect virus virulence. The ts phenotype in the $\mathrm{L}$ segment appeared at passage 4 . The appearance of this ts phenotype was concomitant with the significant reduction in virus virulence, indicating its association with attenuation of virus virulence.

\section{Concluding Remarks}

Results from experiments using mutant RVFVs, obtained by either a classical RNA reassortment approach or RVFV reverse genetics systems and various strains of mice lacking specific gene functions, significantly contributed to the identification of viral proteins that affect RVFV virulence and pathogenicity. These studies revealed that RVFV virulence is under polygenetic control. These results are not surprising, as there is a limitation in the total length of viral genomic RNA segments that the virus can carry. Thus, each viral protein most probably has to have multiple biological functions, including the inactivation of host antiviral responses and promotion of efficient expression of viral proteins or assembly, to exert efficient virus replication/dissemination both in mammalian and mosquito hosts. Several past studies have revealed that the route of virus inoculation, the animal model, the choice of the cell line for preparing the inoculum virus, and the presence of a mosquito component affect the pathogenicity of RVFV. Importantly, accumulating data imply the importance of macrophages in controlling RVFV pathogenicity; thus, inefficient RVFV replication in macro- 
phages has resulted in reduced virus-induced pathogenicity $[6,13,15]$. Moreover, DC-SIGN has been identified as one of the receptors for RVFV, but we still do not know what role DCs play in RVFV pathogenesis. Further studies on response of macrophages and DCs against RVFV infection will be important to understand host and virus interaction at an early stage of infection, which eventually affects the clinical outcome of the disease. We predict that recent progress in various biological approaches, including systems biology and selective suppression of host gene expression, will facilitate further clarification of the mech- anistic functions of RVFV proteins. Additionally, the application of these approaches should yield vital data for better understanding of the disease mechanisms of RVF, including hemorrhagic fever and viral neuroinvasion, as well as development of antivirals.

\section{Acknowledgements}

This work was supported by Public Health Service grant AI101772 from the NIH and a John Sealy Memorial Endowment Fund research grant from The University of Texas Medical Branch.

\section{References}

1 Bird BH, Ksiazek TG, Nichol ST, Maclachlan NJ: Rift Valley fever virus. J Am Vet Med Assoc 2009;234:883-893.

2 Ikegami T, Makino S: The pathogenesis of Rift Valley fever. Viruses 2011;3:493-519.

-3 Nuss JE, Kehn-Hall K, Benedict A, Costantino J, Ward M, Peyser BD, Retterer CJ, Tressler LE, Wanner LM, McGovern HF, Zaidi A, Anthony SM, Kota KP, Bavari S, Hakami RM: Multifaceted proteomic characterization of host protein complement of Rift Valley fever virus virions and identification of specific heat shock proteins, including HSP90, as important viral host factors. PLoS One 2014;9:e93483.

-4 Shieh WJ, Paddock CD, Lederman E, Rao CY, Gould LH, Mohamed M, Mosha F, Mghamba J, Bloland P, Njenga MK, Mutonga D, Samuel AA, Guarner J, Breiman RF, Zaki SR: Pathologic studies on suspect animal and human cases of Rift Valley fever from an outbreak in Eastern Africa, 2006-2007. Am J Trop Med Hyg 2010;83:38-42.

5 Le Coupanec A, Babin D, Fiette L, Jouvion G, Ave P, Misse D, Bouloy M, Choumet V: Aedes mosquito saliva modulates Rift Valley fever virus pathogenicity. PLoS Negl Trop Dis 2013;7:e2237.

6 Gommet C, Billecocq A, Jouvion G, Hasan M, Zaverucha do Valle T, Guillemot L, Blanchet C, van Rooijen N, Montagutelli X, Bouloy M, Panthier JJ: Tissue tropism and target cells of NSs-deleted Rift Valley fever virus in live immunodeficient mice. PLoS Negl Trop Dis 2011;5:e1421.

-7 Hartman AL, Powell DS, Bethel LM, Caroline AL, Schmid RJ, Oury T, Reed DS: Aerosolized Rift Valley fever virus causes fatal encephalitis in African green monkeys and common marmosets. J Virol 2014;88:2235-2245.

8 Smith DR, Bird BH, Lewis B, Johnston SC, McCarthy S, Keeney A, Botto M, Donnelly G, Shamblin J, Albarino CG, Nichol ST, Hensley LE: Development of a novel nonhuman primate model for Rift Valley fever. J Virol 2012; 86:2109-2120.

-9 Reed C, Lin K, Wilhelmsen C, Friedrich B, Nalca A, Keeney A, Donnelly G, Shamblin J,
Hensley LE, Olinger G, Smith DR: Aerosol exposure to Rift Valley fever virus causes earlier and more severe neuropathology in the murine model, which has important implications for therapeutic development. PLoS Negl Trop Dis 2013;7:e2156.

10 Dodd KA, McElroy AK, Jones TL, Zaki SR, Nichol ST, Spiropoulou CF: Rift Valley fever virus encephalitis is associated with an ineffective systemic immune response and activated $\mathrm{T}$ cell infiltration into the CNS in an immunocompetent mouse model. PLoS Negl Trop Dis 2014;8:e2874.

11 Bales JM, Powell DS, Bethel LM, Reed DS, Hartman AL: Choice of inbred rat strain impacts lethality and disease course after respiratory infection with Rift Valley fever virus. Front Cell Infect Microbiol 2012;2: 105.

12 Briant L, Despres P, Choumet V, Misse D: Role of skin immune cells on the host susceptibility to mosquito-borne viruses. Virology 2014;464-465:26-32.

13 Nfon CK, Marszal P, Zhang S, Weingartl HM: Innate immune response to Rift Valley fever virus in goats. PLoS Negl Trop Dis 2012;6: e1623.

14 Morrill JC, Ikegami T, Yoshikawa-Iwata N, Lokugamage N, Won S, Terasaki K, ZamotoNiikura A, Peters CJ, Makino S: Rapid accumulation of virulent Rift Valley fever virus in mice from an attenuated virus carrying a single nucleotide substitution in the M RNA. PLoS One 2010;5:e9986.

15 Kreher F: The Rift Valley fever accessory proteins NSm and P78/NSm-Gn are distinct determinants of virus propagation in vertebrate and invertebrate hosts. Emerg Microbes Infect DOI: 10.1038/emi.2014.71.

16 Won S, Ikegami T, Peters CJ, Makino S: NSm protein of Rift Valley fever virus suppresses virus-induced apoptosis. J Virol 2007;81: 13335-13345.

17 McElroy AK, Nichol ST: Rift Valley fever virus inhibits a pro-inflammatory response in experimentally infected human monocyte derived macrophages and a pro-inflammatory cytokine response may be associated with patient survival during natural infection. Virology 2012;422:6-12.

18 Ermler ME, Yerukhim E, Schriewer J, Schattgen S, Traylor Z, Wespiser AR, Caffrey DR, Chen ZJ, King $\mathrm{CH}$, Gale M Jr, Colonna $\mathrm{M}$, Fitzgerald KA, Buller RM, Hise AG: RNA helicase signaling is critical for type I interferon production and protection against Rift Valley fever virus during mucosal challenge. J Virol 2013;87:4846-4860.

19 Lozach PY, Kühbacher A, Meier R, Mancini R, Bitto D, Bouloy M, Helenius A: DC-SIGN as a receptor for phleboviruses. Cell Host Microbe 2011;10:75-88.

20 Ludwig IS, Lekkerkerker AN, Depla E, Bosman F, Musters RJ, Depraetere S, van Kooyk Y, Geijtenbeek TB: Hepatitis C virus targets DC-SIGN and L-SIGN to escape lysosomal degradation. J Virol 2004; 78:8322-8332.

21 Geijtenbeek TB, Kwon DS, Torensma R, van Vliet SJ, van Duijnhoven GC, Middel J, Cornelissen IL, Nottet HS, KewalRamani VN, Littman DR, Figdor CG, van Kooyk Y: DCSIGN, a dendritic cell-specific HIV-1-binding protein that enhances trans-infection of $\mathrm{T}$ cells. Cell 2000;100:587-597.

22 Smith DR, Steele KE, Shamblin J, Honko A, Johnson J, Reed C, Kennedy M, Chapman JL, Hensley LE: The pathogenesis of Rift Valley fever virus in the mouse model. Virology 2010;407:256-267.

23 de Boer SM, Kortekaas J, de Haan CA, Rottier PJ, Moormann RJ, Bosch BJ: Heparan sulfate facilitates Rift Valley fever virus entry into the cell. J Virol 2012;86:13767-13771.

24 Rippy MK, Topper MJ, Mebus CA, Morrill JC: Rift Valley fever virus-induced encephalomyelitis and hepatitis in calves. Vet Pathol 1992;29:495-502.

25 Gray KK, Worthy MN, Juelich TL, Agar SL, Poussard A, Ragland D, Freiberg AN, Holbrook MR: Chemotactic and inflammatory responses in the liver and brain are associated with pathogenesis of Rift Valley fever virus infection in the mouse. PLoS Negl Trop Dis 2012;6:e1529. 
-26 Reed C, Steele KE, Honko A, Shamblin J, Hensley LE, Smith DR: Ultrastructural study of Rift Valley fever virus in the mouse model. Virology 2012;431:58-70.

-27 Jansen van Vuren P, Tiemessen CT, Paweska JT: Anti-nucleocapsid protein immune responses counteract pathogenic effects of Rift Valley fever virus infection in mice. PLoS One 2011;6:e25027.

28 Couper KN, Blount DG, Riley EM: IL-10: the master regulator of immunity to infection. J Immunol 2008;180:5771-5777.

-29 Dodd KA, McElroy AK, Jones ME, Nichol ST, Spiropoulou CF: Rift Valley fever virus clearance and protection from neurologic disease are dependent on CD4+ $\mathrm{T}$ cell and virus-specific antibody responses. J Virol 2013;87: 6161-6171.

- 30 Schneider H, Weber CE, Schoeller J, Steinmann U, Borkowski J, Ishikawa H, Findeisen P, Adams O, Doerries R, Schwerk C, Schroten $\mathrm{H}$, Tenenbaum T: Chemotaxis of T-cells after infection of human choroid plexus papilloma cells with Echovirus 30 in an in vitro model of the blood-cerebrospinal fluid barrier. Virus Res 2012;170:66-74.

- 31 Hanafi HA, Fryauff DJ, Saad MD, Soliman AK, Mohareb EW, Medhat I, Zayed AB, Szumlas DE, Earhart KC: Virus isolations and high population density implicate Culex antennatus (Becker) (Diptera: Culicidae) as a vector of Rift Valley fever virus during an outbreak in the Nile delta of Egypt. Acta Trop 2011;119:119-124.

- 32 Lihoradova O, Ikegami T: Countermeasure development for Rift Valley fever: deletion, modification or targeting of major virulence factor. Future Virol 2014;9:27-39.

-33 Saluzzo JF, Smith JF: Use of reassortant viruses to map attenuating and temperaturesensitive mutations of the Rift Valley fever virus MP-12 vaccine. Vaccine 1990;8:369375.

34 Billecocq A, Gauliard N, Le May N, Elliott RM, Flick R, Bouloy M: RNA polymerase Imediated expression of viral RNA for the rescue of infectious virulent and avirulent Rift Valley fever viruses. Virology 2008;378:377384
35 Muller R, Saluzzo JF, Lopez N, Dreier T, Turell M, Smith J, Bouloy M: Characterization of clone 13, a naturally attenuated avirulent isolate of Rift Valley fever virus, which is altered in the small segment. Am J Trop Med Hyg 1995;53:405-411.

36 Vialat P, Billecocq A, Kohl A, Bouloy M: The $S$ segment of Rift Valley fever phlebovirus (Bunyaviridae) carries determinants for attenuation and virulence in mice. J Virol 2000; 74:1538-1543.

37 Ikegami T, Won S, Peters CJ, Makino S: Rescue of infectious Rift Valley fever virus entirely from cDNA, analysis of virus lacking the NSs gene, and expression of a foreign gene. J Virol 2006;80:2933-2940.

38 Habjan M, Pichlmair A, Elliott RM, Overby AK, Glatter T, Gstaiger M, Superti-Furga G, Unger H, Weber F: NSs protein of Rift Valley fever virus induces the specific degradation of the double-stranded RNA-dependent protein kinase. J Virol 2009;83:43654375.

39 Mudhasani R, Tran JP, Retterer C, Radoshitzky SR, Kota KP, Altamura LA, Smith JM, Packard BZ, Kuhn JH, Costantino J, Garrison AR, Schmaljohn CS, Huang IC, Farzan M, Bavari S: IFITM-2 and IFITM-3 but not IFITM-1 restrict Rift Valley fever virus. J Virol 2013;87:8451-8464.

40 Bouloy M, Janzen C, Vialat P, Khun H, Pavlovic J, Huerre M, Haller O: Genetic evidence for an interferon-antagonistic function of Rift Valley fever virus nonstructural protein NSs. J Virol 2001;75:1371-1377.

41 Anderson GW Jr, Peters CJ: Viral determinants of virulence for Rift Valley fever (RVF) in rats. Microb Pathog 1988;5:241250.

42 Morrill JC, Jennings GB, Johnson AJ, Cosgriff TM, Gibbs PH, Peters CJ: Pathogenesis of Rift Valley fever in rhesus monkeys: role of interferon response. Arch Virol 1990;110:195212.

43 Garcia MA, Gil J, Ventoso I, Guerra S, Domingo E, Rivas C, Esteban M: Impact of protein kinase PKR in cell biology: from antiviral to antiproliferative action. Microbiol Mol Biol Rev 2006;70:1032-1060.
44 Sen A, Pruijssers AJ, Dermody TS, GarciaSastre A, Greenberg HB: The early interferon response to rotavirus is regulated by $\mathrm{PKR}$ and depends on MAVS/IPS-1, RIG-i, MDA-5, and IRF3. J Virol 2011;85:3717-3732.

45 Schulz O, Pichlmair A, Rehwinkel J, Rogers NC, Scheuner D, Kato H, Takeuchi O, Akira S, Kaufman RJ, Reis e Sousa C: Protein kinase $\mathrm{R}$ contributes to immunity against specific viruses by regulating interferon mRNA integrity. Cell Host Microbe 2010;7:354-361.

46 Kalveram B, Lihoradova O, Indran SV, Lokugamage N, Head JA, Ikegami T: Rift Valley fever virus NSs inhibits host transcription independently of the degradation of dsRNAdependent protein kinase PKR. Virology 2013;435:415-424.

47 Bird BH, Khristova ML, Rollin PE, Ksiazek TG, Nichol ST: Complete genome analysis of 33 ecologically and biologically diverse Rift Valley fever virus strains reveals widespread virus movement and low genetic diversity due to recent common ancestry. J Virol 2007;81: 2805-2816.

48 Terasaki K, Won S, Makino S: The C-terminal region of Rift Valley fever virus NSm protein targets the protein to the mitochondrial outer membrane and exerts antiapoptotic function. J Virol 2013;87:676-682.

49 Won S, Ikegami T, Peters CJ, Makino S: NSm and 78-kilodalton proteins of Rift Valley fever virus are nonessential for viral replication in cell culture. J Virol 2006;80:8274-8278.

50 Bird BH, Albarino CG, Nichol ST: Rift Valley fever virus lacking NSm proteins retains high virulence in vivo and may provide a model of human delayed onset neurologic disease. Virology 2007;362:10-15.

-51 Kading RC, Crabtree MB, Bird BH, Nichol ST, Erickson BR, Horiuchi K, Biggerstaff BJ, Miller BR: Deletion of the NSm virulence gene of Rift Valley fever virus inhibits virus replication in and dissemination from the midgut of Aedes aegypti mosquitoes. PLoS Negl Trop Dis 2014;8:e2670.

52 Weingartl HM, Zhang S, Marszal P, McGreevy A, Burton L, Wilson WC: Rift Valley fever virus incorporates the $78 \mathrm{kDa}$ glycoprotein into virions matured in mosquito $\mathrm{C6} / 36$ cells. PLoS One 2014;9:e87385. 